\title{
Impact of Mole Mapping in the Italian Health System
}

\author{
Ignazio Stanganelli ${ }^{a}$ Paolo Ascierto ${ }^{b}$ Riccardo Bono ${ }^{c}$ Vincenzo De Giorgi ${ }^{d}$ \\ Nicola Pimpinelli $^{d}$ Vanna Chiarion-Sileni ${ }$ Giuseppe Palmieri ${ }^{f}$ \\ Maria Antonietta Pizzichettag ${ }^{9}$ Alessandro Testori ${ }^{\text {h }}$

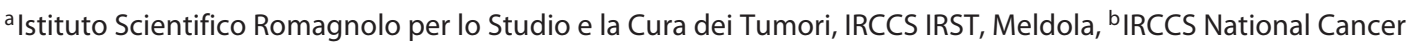 \\ Institute, Naples, 'Immacolata Dermatological Institute, IRCCS, Rome, ${ }^{d}$ Dermatologic Clinic, University of Florence, \\ Florence, ${ }^{e}$ Veneto Institute of Oncology, Padua, ${ }^{\mathrm{f}}$ Genetica Oncologica, CNR, Sassari, ${ }^{9}$ Centro di Riferimento \\ Oncologico, Aviano, and ${ }^{\mathrm{h}}$ Istituto Europeo di Oncologia, Milan, Italy
}

\section{Key Words}

Melanoma $\cdot$ Melanocytic nevus $\cdot$ Mole mapping $\cdot$ Digital epiluminescence microscopy $\cdot$ Dermoscopy $\cdot$ Survey

\begin{abstract}
Objective: To analyze routine clinical management of atypical melanocytic lesions through monitoring or surgery in Italian hospitals. Methods: A nationwide survey of clinical practices was conducted. Results: Digital monitoring is performed in most Italian hospitals and is preferred over excision for single atypical melanocytic lesions in $82 \%$ of hospitals. For multiple atypical lesions, $60 \%$ of high-volume hospitals prefer digital monitoring to surgical excision (40\%). There is a statistically significant difference between highand low-volume hospitals ( 60 vs. $39 \% ; p=0.003$ ). Digital monitoring is performed at mean intervals of $4 / 5$ months for both types of lesions. Conclusions: We show an asymmetric relation between application of the method and practical impact based on available clinical evidence. Although digital monitoring provides better characterization of the evolution of melanocytic lesions, our results indicate that the advantages and limitations of this method require further investigation.

Copyright $\odot 2013$ S. Karger AG, Basel
\end{abstract}

\section{Introduction}

Digital dermoscopy has opened new perspectives in the management of patients at risk for melanoma, in the monitoring of melanocytic lesions and in applied research [1]. Digital systems provide computer storage of clinical and dermoscopic images of nevi, facilitating detailed followup of geometric, chromatic and structural modifications of individual melanocytic lesions. Images are generally obtained using a video dermatoscope, but digital cameras and digital stereo microscopes may be used as well.

Digital monitoring, commonly referred to as 'mole mapping', is used to monitor atypical melanocytic lesions lacking melanoma-specific criteria. Comparisons are made at intervals of 3-6 months and changes evaluated to identify featureless melanomas or nevus incipiens $[2,3]$. Long-term monitoring at intervals $>6$ months is used in cases of multiple atypical nevi in patients at high risk for melanoma [4-10].

We analyzed the use of digital monitoring in Italian hospitals, with the goal of evaluating the impact of digital monitoring and the management of single or multiple suspicious melanocytic lesions using either digital dermoscopic follow-up or surgical removal in routine clinical practice.

\section{KARGER}

E-Mail karger@karger.com

www.karger.com/drm
(C) 2013 S. Karger AG, Basel

$1018-8665 / 13 / 2265-0013 \$ 38.00 / 0$
Maria A. Pizzichetta, MD

Division of Medical Oncology C, Preventive Oncology

Centro di Riferimento Oncologico, IRCCS

Via Franco Gallini 2, IT-33081 Aviano (Italy)

E-Mail pizzichetta@cro.it 
Table 1. Indication for digital monitoring of atypical melanocytic nevi in Italian hospitals grouped according to yearly melanoma diagnoses into high-volume $(>25)$ and low-volume $(\leq 25)$ centers

\begin{tabular}{llll}
\hline Type of lesion & \multicolumn{2}{l}{ Type of center } \\
\cline { 2 - 4 } & $\begin{array}{l}\text { high-volume } \\
(\mathrm{n}=56)\end{array}$ & $\begin{array}{l}\text { low-volume } \\
(\mathrm{n}=62)\end{array}$ & $\begin{array}{l}\text { all } \\
(\mathrm{n}=118)\end{array}$ \\
\hline Single & $79 \%$ & $86 \%$ & $82 \%$ \\
Multiple & $60 \%$ & $39 \% *$ & $52 \%$ \\
\hline
\end{tabular}

$* \mathrm{p}=0.003$.

\section{Methods}

Briefly, a nationwide survey of clinicians responsible for the diagnosis, therapy or follow-up phases of melanoma care in Italian hospitals was conducted. Italian hospitals with $\geq 200$ beds $(n=285)$ were subdivided into 145 hospitals with $200-399$ beds and 140 hospitals with $\geq 400$ beds and a proportionally stratified random sample ( $n=120$ centers), stratified by number of beds and geographic distribution, was selected. Two or three clinicians were interviewed at each center, resulting in approximately 250 interviews and a predicted margin of error - $95 \%$ confidence level - of $7.7 \%$.

Based on the findings, centers were grouped by number of new melanoma diagnoses per year into low- and high-volume centers, around the median value of 25 . Variables were analyzed in the total sample/total Italian hospitals, and comparisons were made between high- and low-volume centers using Pearson's $\chi^{2}$ test and the zeta test at $95 \%$ confidence level. Detailed methods are presented elsewhere in this issue [11].

\section{Results}

Digital monitoring is performed in most Italian hospitals. Management of single atypical melanocytic lesions was carried out with digital monitoring in $82 \%$ of hospitals and this was similar regardless of the volume of melanoma patients treated. Overall, surgical excision was used in $18 \%$ of hospitals, and results did not differ statistically between high-volume centers ( $>25$ melanoma diagnoses/year) and low-volume centers ( $\leq 25$ diagnoses/ year). Follow-up was performed after an average of $4 / 5$ months in both groups of hospitals.

In case of multiple atypical lesions, different approaches are used in the two groups of hospitals. Among highvolume centers, $60 \%$ use monitoring and $40 \%$ opt for surgery. In contrast, in low-volume centers the ratio is inverted, with 39\% using digital monitoring and $61 \%$ choosing surgical excision of multiple atypical lesions.
The difference in the use of monitoring between highand low-volume centers was statistically significant ( $\mathrm{p}=$ 0.003) (table 1). Digital monitoring of multiple atypical lesions was performed after an average of $4 / 5$ months.

\section{Discussion}

Digital monitoring, more commonly referred to as mole mapping, is used to monitor atypical melanocytic lesions that do not meet the criteria for melanoma. It involves evaluation and comparison of short-term changes (3-6 months) or long-term changes ( $>6$ months). So far it has not been possible to assess the accuracy of this technique.

In practice, although there are no unequivocal dermoscopy criteria for defining a 'lesion to store for followup', melanocytic nevi with the highest melanoma potential are (1) nevus with eccentric hyperpigmentation, (2) nevus with eccentric hypopigmentation, (3) melanocytic nevus with homogeneous black pattern (no corneal lamellae) and (4) nevus with multifocal hypo-/hyperpigmentation [12-15].

According to the Italian guidelines and those issued by the International Dermoscopy Society, frequent dermoscopic monitoring (3-6 months) is recommended for flat atypical melanocytic lesions, and any change is an indication for surgical removal $[16,17]$.

The International Dermoscopy Society guidelines suggest that frequent mole mapping can be used to monitor (1) pigmented lesion in a high-risk patient with a reported or documented history of change depending on clinical and/or dermoscopic appearance, (2) melanocytic lesions with a symmetrical peripheral rim of globules with an asymmetry of structures within the lesion, (3) atypical blue nevi, (4) isolated pigmented or atypical seborrheic keratosis, (5) acral melanocytic lesions in adults with atypical clinical and/or 'non-typical' dermoscopy patterns, (6) dermatofibromas with atypical dermoscopic patterns and (7) lesions with uncertain diagnosis at clinical and/or dermoscopic examination [17]. However, surgical excision is strongly recommended when a single atypical lesion (a clinical-dermoscopic 'ugly duckling') has reticular, globular and homogeneous structures and eccentric hyperpigmentation or a clear clinical history of recent changes [15-18].

Digital follow-up is not useful for nodular lesions with atypical features, as it is impossible to exclude a diagnosis of nodular melanoma $[16,17]$. Patients undergoing periodic examinations generally have many nevi and one pre- 
Table 2. Advantages and limitations of mole mapping

Advantages
Identification of changes in dermoscopic characteristics in featureless melanomas or detection
at an early stage
at an early stage

Reference

Altamura et al. (2008) [3]

Argenziano et al. (2008) [22]

Haenssle et al. (2006) [7]

Bauer et al. (2005) [8]

Skvara et al. (2005) [21]

Robinson and Nickoloff (2004) [9]

Malvehy and Puig (2002) [6]

Menzies et al. (2001) [2]

Kittler et al. (2000) [4]

Reduced number of unnecessary excisions

Fuller et al. (2007) [5]

Bauer et al. (2005) [8]

Robinson and Nickoloff (2004) [9]

Malvehy and Puig (2002) [6]

Elevated compliance with short-term follow-up

Argenziano et al. (2008) [22]

Bauer et al. (2005) [8]

Self-examination education

Robinson and Nickoloff (2004) [9]

Schiffner et al. (2003) [10]

Identification of changes in dermoscopic features at follow-up, particularly in dysplastic nevi

Particularly useful in high-risk patients

Fuller et al. (2007) [5]

Haenssle et al. (2006) [7]

Increased sensitivity in the recognition of melanoma at follow-up

Haenssle et al. (2006) [7]

Identification of small-diameter melanomas with less visible scars

Robinson and Nickoloff (2004) [9]

A better relationship between patient and heath care operators

Integration of total body photography

Limitations

Robinson and Nickoloff (2004) [9]

Malvehy and Puig (2002) [6]

Reference

Time-consuming (30-60 min per patient)

Haenssle et al. (2006) [7]

Robinson and Nickoloff (2004) [9]

Malvehy and Puig (2002) [6]

Menzies et al. (2001) [2]

Risk of low compliance due to long-term follow-up

Argenziano et al. (2008) [22]

Haenssle et al. (2006) [7]

Schiffner et al. (2003) [10]

Extreme subjectivity in determining the follow-up period, features of patients and lesions to be Carli et al. (2006) [23]

evaluated (subjective parameters)

Malvehy and Puig (2002) [6]

Images intended for comparison may contain artifactual changes caused by operator errors or Altamura et al. (2008) [3]

skin changes other than those of interest

Follow-up is limited to high-risk patients

Argenziano et al. (2008) [22]

Bauer et al. (2005) [8]

Robinson and Nickoloff (2004) [9]

In slow-growing melanomas (e.g. lentigo maligna of the face) follow-up after 3 months is not Altamura et al. (2008) [3]

long enough to detect changes of the dermoscopic features; more follow-ups are required

Follow-up at 6 weeks does not present an advantage over follow-up at 3 months

Need for dermoscopic examination of all lesions at follow-up examinations, not only the previously selected lesions

Absence of guidelines

Altamura et al. (2008) [3]

Fuller et al. (2007) [5]

Carli et al. (2006) [23]

Risk of not excising lesions highly suspicious for melanoma at first examination

Haenssle et al. (2006) [7]

Expert health operators

Bauer et al. (2005) [8]

Elevated cost-benefit

Schiffner et al. (2003) [10] 
dominant type of atypical nevus. In these patients, the presence of a lesion that does not belong to the predominant type of atypical nevi requires special attention, particularly when no other atypical nevi show similar dermoscopic features. Diagnostic and therapeutic procedures in these patients therefore depend on the number of atypical nevi and the atypical nevus variant.

From a practical point of view, digital image storage to monitor changes over time should be limited to selected cases in whom the clinician has adequately weighed the potential benefits (avoiding unnecessary biopsy) against the risks (leaving a possible melanoma in situ). The possibility that a patient may not turn up for the subsequent follow-up should also be taken into account [10, 16-23]. However, it is clear that melanocytic lesions with features of nevus-like melanoma can benefit from short-term digital monitoring [21].

The advantages and limitations of digital monitoring have been shown in numerous studies (table 2). Our results seem to contradict what appears to be the current application of digital monitoring. Although we found a mean monitoring frequency of $4 / 5$ months, which falls between short-term follow-up (3 months) and long-term follow up ( $>6$ months) and is in line with published recommendations $[14,16]$, we also found that management of atypical melanocytic lesions does not always conform to recommendations. (1) A single atypical lesion, a poten- tial 'ugly duckling', is left in situ more often ( $82 \%$ of hospitals) than multiple atypical lesions (52\% of hospitals). (2) Multiple melanocytic lesions, which are candidates for digital monitoring that could identify a potential featureless melanoma and at the same time reduce the number of 'needless' excisions, are instead treated with surgical excision in $48 \%$ of the hospitals sampled.

This seemingly paradoxical approach (monitoring for a single atypical lesion but surgical excision for multiple atypical lesions) may confirm results of an Italian multicenter study that revealed a lack of clear and standardized inclusion and exclusion criteria for digital follow-up [23]. Moreover, our results show asymmetry between application of the method and practical impact based on the evidence currently available in clinical practice.

Although digital monitoring permits better characterization of the evolution of a melanocytic lesion, the results of this study show that the advantages and limitations of this method require further investigation.

\section{Disclosure Statement}

The authors received no funding and report no conflict of interest.

\section{References}

1 Massone C, Di Stefani A, Soyer HP: Dermoscopy for skin cancer detection. Curr Opin Dermatol 2005; 17:147-153.

-2 Menzies SW, Gutenev A, Avramidis M, Ba$\operatorname{trac} \mathrm{A}, \mathrm{McCarthy}$ WH: Short-term digital surface microscopic monitoring of atypical or changing melanocytic lesions. Arch Dermatol 2001;137:1583-1589.

-3 Altamura D, Avramidis M, Menzies SW: Assessment of the optimal interval for and sensitivity of short-term sequential digital dermoscopy monitoring for the diagnosis of melanoma. Arch Dermatol 2008;144:502-506.

-4 Kittler $\mathrm{H}$, Pehamberger $\mathrm{H}$, Wolff $\mathrm{K}$, Binder M: Follow-up of melanocytic skin lesions with digital epiluminescence microscopy: patterns of modifications observed in early melanoma, atypical nevi, and common nevi. J Am Acad Dermatol 2000;43:467-476.

-5 Fuller SR, Bowen GM, Tanner B, Florell SR, Grossman D: Digital dermoscopic monitoring of atypical nevi in patients at risk for melanoma. Dermatol Surg 2007;33:1198-1206.
6 Malvehy J, Puig S: Follow-up of melanocytic skin lesions with digital total-body photography and digital dermoscopy: a two-step method. Clin Dermatol 2002;20:297-304.

7 Haenssle HA, Krueger U, Vente C, Thoms KM, Bertsch HP, Zutt M, Rosenberger A, Neumann C, Emmert S: Results from an observational trial: digital epiluminescence microscopy follow-up of atypical nevi increases the sensitivity and the chance of success of conventional dermoscopy in detecting melanoma. J Invest Dermatol 2006;126:980-985.

8 Bauer J, Blum A, Strohhäcker U, Garbe C: Surveillance of patients at high risk for cutaneous malignant melanoma using digital dermoscopy. Br J Dermatol 2005;152:87-92.

9 Robinson JK, Nickoloff BJ: Digital epiluminescence microscopy monitoring of high-risk patients. Arch Dermatol 2004;140:49-56.

10 Schiffner R, Schiffner-Rohe J, Landthaler M, Stolz W: Long-term dermoscopic follow-up of melanocytic naevi: clinical outcome and patient compliance. Br J Dermatol 2003;149: 79-86.
11 Mingozzi E, Fregosi S, Gandini S, Stanganelli I, Chiarion-Sileni V, Testori A: Melanoma Task Force (META) project in Italy: methodology. Dermatology 2013;226(suppl 1): $1-2$.

12 Hofmann-Wellenhof R, Blum A, Wolf IH, Piccolo D, Kerl H, Garbe C, Soyer HP: Dermoscopic classification of atypical melanocytic nevi (Clark nevi). Arch Dermatol 2001; 137:1575-1580.

13 Kenet RO, Fitzpatrick TB: Reducing mortality and morbidity of cutaneous melanoma: a six year plan. B). Identifying high and low risk pigmented lesions using epiluminescence microscopy. J Dermatol 1994;21:881-884.

14 Blum A, Soyer HP, Garbe C, Kerl H, Rassner G, Hofmann-Wellenhof R: The dermoscopic classification of atypical melanocytic naevi (Clark naevi) is useful to discriminate benign from malignant melanocytic lesions. Br J Dermatol 2003;149:1159-1164. 
15 Pizzichetta MA, Stanganelli I, Bono R, Soyer HP, Magi S, Canzonieri V, Lanzanova G, Annessi G, Massone C, Cerroni L, Talamini R; Italian Melanoma Intergroup (IMI): Dermoscopic features of difficult melanoma. Dermatol Surg 2007;33:91-99.

16 Chimenti S, Argenziano, Di Stefani A, Andreassi L, Carli P, de Giorgi V, Ferrara G, Ferrari A, Gasparini S, Giovene GL, et al: Guidelines in dermoscopy. G Ital Dermatol Venereol 2005; 140:329-347.

17 Bowling J, Argenziano G, Azenha A, Bandic J, Bergman R, Blum A, et al: Dermoscopy key points: recommendations from the international dermoscopy society. Dermatology 2007;214:3-5.
18 Arevalo A, Altamura D, Avramidis M, Blum A, Menzies S: The significance of eccentric and central hyperpigmentation, multifocal hyper/hypopigmentation, and the multicomponent pattern in melanocytic lesions lacking specific dermoscopic features of melanoma. Arch Dermatol 2008;144:1440-1444.

19 Carli P, De Giorgi V, Giannotti B: Dermoscopy and early diagnosis of melanoma. The light and the dark. Arch Dermatol 2001;137: 1641-1644.

20 Kittler H, Binder M: Risks and benefits of sequential imaging of melanocytic skin lesions in patients with multiple atypical nevi. Arch Dermatol 2001;137:1590-1595.

21 Skvara H, Teban L, Fiebiger M, Binder M, Kittler H: Limitations of dermoscopy in the recognition of melanoma. Arch Dermatol 2005;141:155-160.
22 Argenziano G, Mordente I, Ferrara G, Sgambato A, Annese P, Zalaudek I: Dermoscopic monitoring of melanocytic skin lesions: clinical outcome and patient compliance vary according to follow-up protocols. Br J Dermatol 2008;159:331-336.

23 Carli P, Ghigliotti G, Gnone M, Chiarugi A, Crocetti E, Astorino S, Berti UA, Broganelli P, Carcaterra A, Corradin MT, Pellacani G, Piccolo D, Risulo M, Stanganelli I, De Giorgi V: Baseline factors influencing decisions on digital follow-up of melanocytic lesions in daily practice: an Italian multicenter survey. J Am Acad Dermatol 2006;55:256-262. 